\title{
Vision Based Self-Guided Quadcopter Landing on Moving Platform During Fault Detection
}

\author{
Peni Jitoko ${ }^{\mathrm{a}}$, Epeli Kama ${ }^{\mathrm{b}}$, Utkal Mehta ${ }^{\mathrm{c}}$ and Aneesh A. Chand ${ }^{\mathrm{d}}$ \\ ${ }^{\text {a-d}}$ Electrical and Electronics Engineering, The University of the South Pacific (USP), Laucala Campus, Suva, \\ Fiji Islands. \\ peni@skywardindustries.com ${ }^{\mathrm{a}}$, epeli.kama@waf.com.fj ${ }^{\mathrm{b}}$, utkal.mehta@usp.ac.fj ${ }^{\mathrm{c}}$, aneeshamitesh@gmail.com ${ }^{\mathrm{d}}$ \\ Corresponding author: *utkal.mehta@usp.ac.fj
}

\begin{abstract}
Fault occurrence in the quadcopter is very common during operation in the air. This paper presents a real-time implementation to detect the fault and then the system is guaranteeing to safely land on the surface, even the moving landing platform. Primarily, PixHawk auto-pilot was used to verify in real-time, with platform detection and various environmental conditions. The method is ensuring the quadcopter operates in the landing area zone with the help of a GPS feature. Then the precise landing on the astable-landing platform is calibrated automatically using the vision-based learning feedback technique. The proposed objective is developed using reconfigurable Raspberry Pi-3 with a Pi camera. The full decision on an efficient landing algorithm is deployed into the quadcopter. The system is self-guided and automatically returns to home-based whenever the fault detects. The study is conducted with the situation of low battery operation and the trigger of auto-pilot helps to land the device safely before any mal-function. The system is featured with predetermined speed and altitude while navigating the home base, thus improves the detection process. Finally, the experiment study provided successful trials to track usable platform, landing on a restricted area, and disarm the motors autonomously.
\end{abstract}

Keywords: Quadcopter, fault detection, autonomous landing, image processing.

\section{Introduction}

Today, unmanned aerial vehicles (UAVs) have progressed dramatically in many, namely, hovering multi-copters, fixed-wing designs, real-time stability and control algorithms, sensor technology, and automated waypoint navigation 1]. The quadcopter is one of the UAVs, have been utilized several applications for surveillance purposes 2], package delivery 3], mapping 4], agricultural applications 1,5-6], security 2], and also media recording 7]. For any application bases on UAVs, the most critical process is vertical take-off and landing which requires an operation of a multi-channel radio controller. This process can be automated with the recent development of advanced open-source flight controllers such as PixHawk 8], Pixracer 9], Erle-Brain Linux Autopilot 10], and PXFmini Autopilot Shield 11]. It is important to note that, any of these flight controllers are programmable with a processor. Even during the flight of the quadcopter, unusual and unexpected events such as actuator failure, sensor failure, and structural failure may occur 11]. The safe flight of aircraft depends on real-time online Fault Detection and Identification (FDI) of the abnormal state of the quadcopter. Analytical 
model-based methods, knowledge-based methods, and signal processing-based methods are the three types of fault diagnosis methods currently available for UAVs. This resarch tries to use the PixHawk flight controller, which uses a 32bit STM32F427 Cortex M4 core processor chip, and to automate the landing process of a quadcopter by incorporating embedded solution with image processing that is within the scope of the objective.

Interestingly, computer-vision-based UAVs are gaining importance as they are cheap, small in size, more compact visual sensors, and have shown great benefits in the field of UAV navigation. A computer vision system onboard UAV is a typically equipped with sensors such as Global Positioning System (GPS) and Inertial Navigation Sensors (INS) are common tracking methods. Autonomous landing is a sequential task and is well suited for vision-based state estimation and control has recently been an active topic of research. Studies have revealed that there are different types of real-time vision systems for a rotor-craft UAV that was introduced. A design was developed with proper estimation with speed relative to the known landing target at $30 \mathrm{~Hz} 12]$. Customized cimputer vision algorithms and off-the-shelf hardware are used in the visual system to perform real-time image processing, segmentation, feature point extraction, camera control, and both linear and nonlinear optimization for model-based pose estimation. Similar features were also well-integrated in UAVs 13], smart path guidance mobile aid 14] and efficient two-fingured gripper 15]. Togather with this, the motion planning and control is always essential for various applications including manipulators 16].

Current literature reported that autonomous UAV navigation involves the acquisition and the analysis of data either received or transmitted or implementation of UAV. It also depends on the environment the UAV is exposed to and how data is being interfered with depending on the environment. Sensing, state estimation, perception, and situational awareness are the four core functions of a navigation device, from lowest to the highest level. Regarding the four functions, different types of sensors such as the Global Navigation Satellite System (GNSS), laser range scanners (LRFs), monocular cameras, stereo cameras, and the newly populated RGB-D (Red Green Blue-Digital) sensors have been explored. With respect to solving the landing problem, most previous researches rely on on-board sensors where experiments have been conducted in both indoor- and outdoor- environments 13]. Certainly, there is a limitation with PI control system for positioning application 17]. Guo et al. 18], presented method to detect the sensor faults using the proportional and multiple integral fault detecton filter. Similarly, Alexis et al. 19] and Feng et al. 20], have developed autonomous landing of UAV 
using model predictive control.

To achive real-time online FDI, there are different mrthod developed. Knowledge-based FDI approaches primarily rely on the expertise of specialists in the field to develop programs. Device FDI can be accomplished by simulating expert reasoning processes. Expert system-based FDI 21], fuzzy inference 22], and fault trees 23] are examples of such approaches. Fuzzy logic-based control schemes were presented for landing purposes 24,25$]$. The efficacy of the controlling any unstable system, a good mathematical model is essential. For developoing accurate models, the common sensors available on the quadcopter are required to include in the data collection. Chen et al. 26], suggested an adaptive observer-based quadcopter fault diagnosis approach based on a mathematical model of quadcopter UAVs. The simulation results demonstrate that the built robust nonlinear controller and fault estimation scheme are efficient.

One of the important components of any UAVs is rechargeable batteries. Most UAVs are designed with a rechargeable lithium-polymer (LiPo) battery, which has a higher energy capacity than other battery types but has a low flight endurance of about 10-30 minutes at best 20]. A variety of approaches have been proposed to address these issues, including the integration of a tether for power and data transmission 27], autonomous deployment and recovery from a charging station 28], solar-powered photovoltaic (PV) panels 29], and the invention of high-power density batteries 30]. Though the quadcopter has several capabilities it is limited by the energy supplied from the batteries. The operation time in the air is limited by the payload and quality of the batteries. It has been found that normal flight time is bare a few minutes up to 30 minutes in a controlled environment. It is desired to operate UAVs continuously longer flight time but it requires to charge on time or replace with spare batteries. To avoid damages and failing the system in general, manual attention is required to land the system manually from time to time and check or replace the battery terminal voltage 31 ]. A technique was presented for landing a VTOL UAV considering again well-known issue of moving platform using optical flow 13]. Kong et al. 32], propsed infrared stereo based vision system to land UAVs automatically. Despite the number of developed strategies and smart devices, the landing issue is still an open problem to solve efficient ways. The common navigation system uses gyroscopes, accelerometers and magnetometers to provide information about the attitude of the vehicle. However, these are highly nonlinear in nature and changes with accelerations.

The paper attempts is to developing a low cost solution, which can improve the handling and landing 
issues of quadcopter following general safety standards of the international drone community. The quadcopter system was developed using PixHawk auto-pilot and all other related programing software. A smart vision based tracking system was developed to integrate into the system. The solution is simple yet effective for the precise landing and to locate the home area. The landing accuracy was achieved upto range of about $10 \mathrm{~cm}$ of the center target landing spot and have a high initial repeatability percentage of $80 \%$ which can be improved over the time. The conclusions and future scope have been derived at the end.

\section{System Overview}

In this section, we introduce a prototype modeled with a self-guided quadcopter, including each of the features, and procedures were undertaken to complete the project effectively.

\subsection{Protoypes Model}

The quadcopter was assembled with a PixHawk flight controller in this research, as given in Figure 1. The establishment of a quadcopter was based on the following:

- DJI F450 Flame Wheel Body Frame

- DJI Tuned Propulsion System

- DJI F450 Landing Gear

- PixHawk Flight Controller (auto-pilot)

- Raspberry Pi 3 (Companion Computer)

- Raspberry Pi Camera version 2

- $\quad$ Turnigy 8 Channel Radio Controller $2.4 \mathrm{GHz}$

- Wireless Radio Telemetry $415 \mathrm{MHz}$

- Ublox-Neo-M8N GPS/Compass

- PixHawk Power Module

- $\quad$ Lithium-Polymer Batteries (16.4 V) 


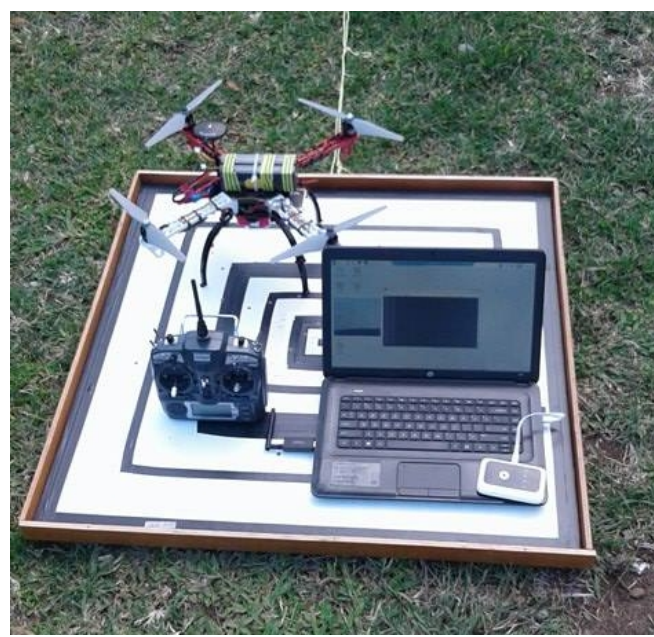

Figure 1. Vission based self-guided quadcopter

\subsection{Ground Control Station}

The most commonly used Mission Planner (MP) software and PixHawk auto-pilot are used to wirelessly monitor the quadcopter during flight. Figure 2 shows that autonomous way-point navigation planning on MP, with this feature the quadcopter can autonomously fly to way-points of the user's inputs.

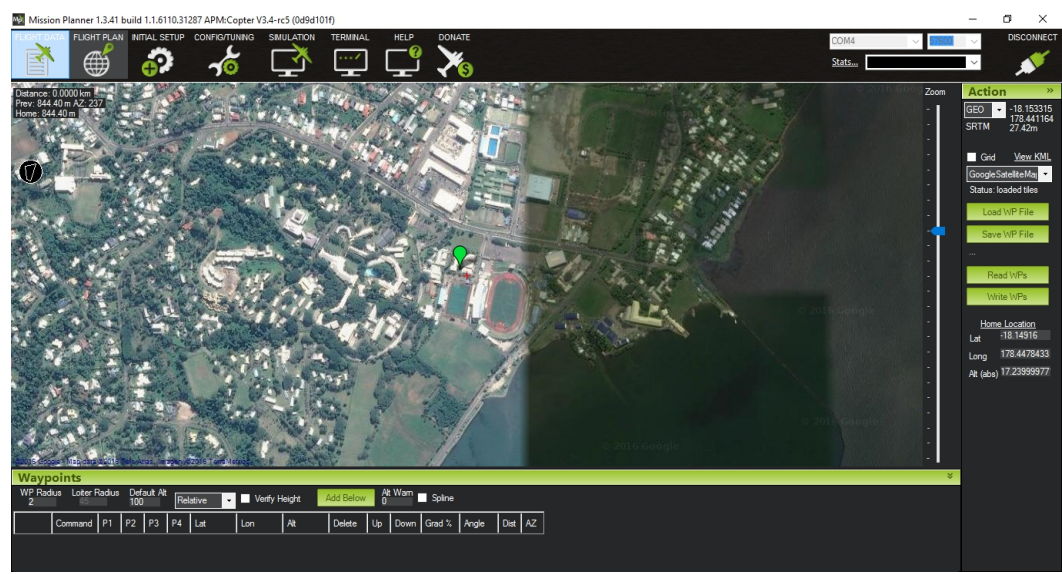

Figure 2. Navigation planning map

\subsection{Quadcopter System Tuning and Flight Mode Tests}

After the successful assembly and installation of the necessary hardware and software, it is required to tune the Radio Controller, Control System parameters (PID), compass, gyroscope, and accelerometer to give the quadcopter a more stable and responsive flight and also give accurate readings from the sensors for analysis under the data log feature in MP. In addition, flight modes and various parameters are required to set up into flight modes such as Stabilize, Loiter, RTL (Return to Launch), ALT-HOLD (Altitude Hold), and Land Mode. 


\subsection{Image and Video Processing/Landing Platform Detection}

Image and video processing is done on-board quadcopter using a Raspberry Pi 3 (Companion Computer) and a Pi Camera version 2. The technique requires developing an elegant solution towards detecting the landing platform and therefore suitable libraries such as Open CV is downloaded into the Pi.

\subsection{Raspberry Pi and PixHawk Communication}

Once the video processing program has been set up with its constraints with regards to processing speed, the next step is to set the communication between the Raspberry Pi and PixHawk. Physically the Raspberry Pi 3 and the PixHawk communicates through the TX-RX pins on the pi connected to the telemetry port 2 on the PixHawk, the two boards communicate using the MavLink Protocol. A library called Drone-Kit was downloaded into the Pi which contained all the various dependencies for Pi and PixHawk communication.

\subsection{Precision Landing On a Stationary and Moving Platform}

One of the latest features of the PixHawk-autopilot is called precision landing, this is a function, which allows any multi-rotor to perform an autonomous landing precisely on a landing target. This function is sub-divided into two features, the precision landing feature can either be used with an IR-LOCK beacon or with a Companion Computer, the project team works with the companion computer method (Raspberry-pi 3), this function (precision landing) is only activated when the quadcopter is in LAND mode. The information required by the precision landing function is the image resolution, horizontal and vertical field of view of the pi-camera, and center coordinates of the landing platform within the image taken.

It is best to test first manually by invoking the LAND command mode from the radio controller, which in turn invokes the precision landing process if the landing platform is detected. Hence, the quadcopter would take off manually, a red LED attached to the quadcopter might light up to indicate whether the platform detected. Then after the quadcopter can be switched to land mode to invoke the precision landing function.

Numerous tests were conducted under various sunlight and wind conditions to see how the quadcopter would react and its reliability in the landing process. Another objective of the work to incubate features with slow battery scenario. A low battery fail-safe voltage is set, the quadcopter is flown off to 
any random location manually but when the fail-safe is activated upon detecting low battery voltage, RTL (Return to Launch) mode is automatically invoked. The quadcopter returns to the Home location which is where the quadcopter is originally armed to fly (Landing Platform). During RTL mode the quadcopter fly's up $15 \mathrm{~m}$ and heads toward the Home location.

Once at home location, the quadcopter is not directly above the landing platform but with a few meters off-set due to GPS inaccuracies, it will hover for 5 seconds giving time for the landing platform program to find the landing platform, once the landing platform is detected by the Pi. It will automatically invoke Land mode, thus allowing the precision landing process to take over. Tests on a moving landing platform were also done, in both stationary and moving landing platforms but there are limitations.

\section{Implemented quadcopter system with proposed technique}

In this section, a discussion will be made on the overall quadcopter hardware system. Figure 3 gives an overview of the quadcopter system hardware architecture, developed in this work.

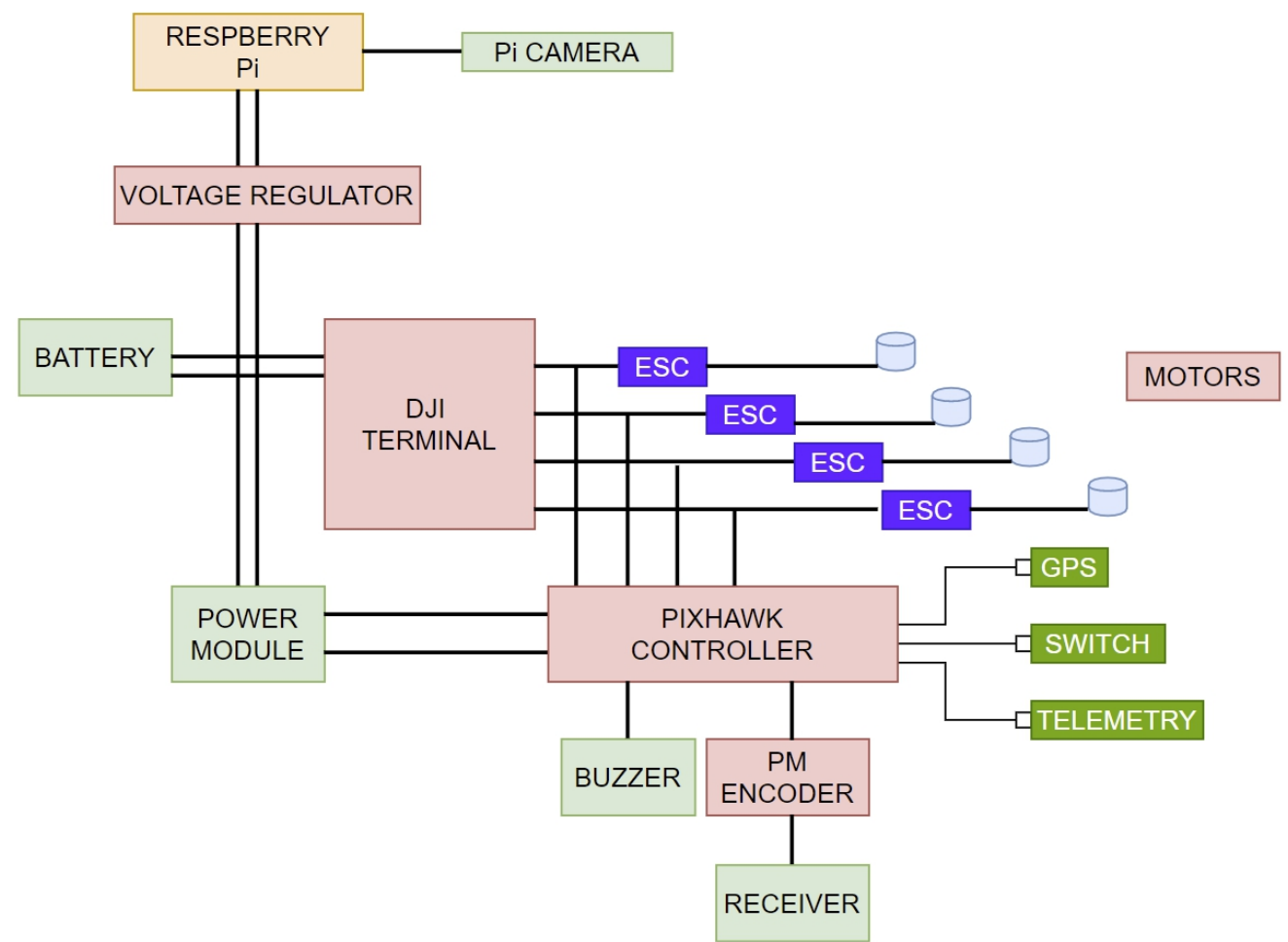

Figure 3. Proposed quadcopter overall block-diagram

The MP is helped to communicate with a telemetry system with a frequency of operation $433 \mathrm{MHz}$. The ground control station is given in Figure 4 and Figure 5, with the actual operating environment. The GCS allows the user to monitor the quadcopter battery status, attitude, and location. With a 
revised feature, the system allows users to send commands and changes parameters remotely, such as the flight altitude and speed. Nowadays, the method is possible to adopt using the reconfigurable and reprogrammable controller such as FPAA for position control system 14].

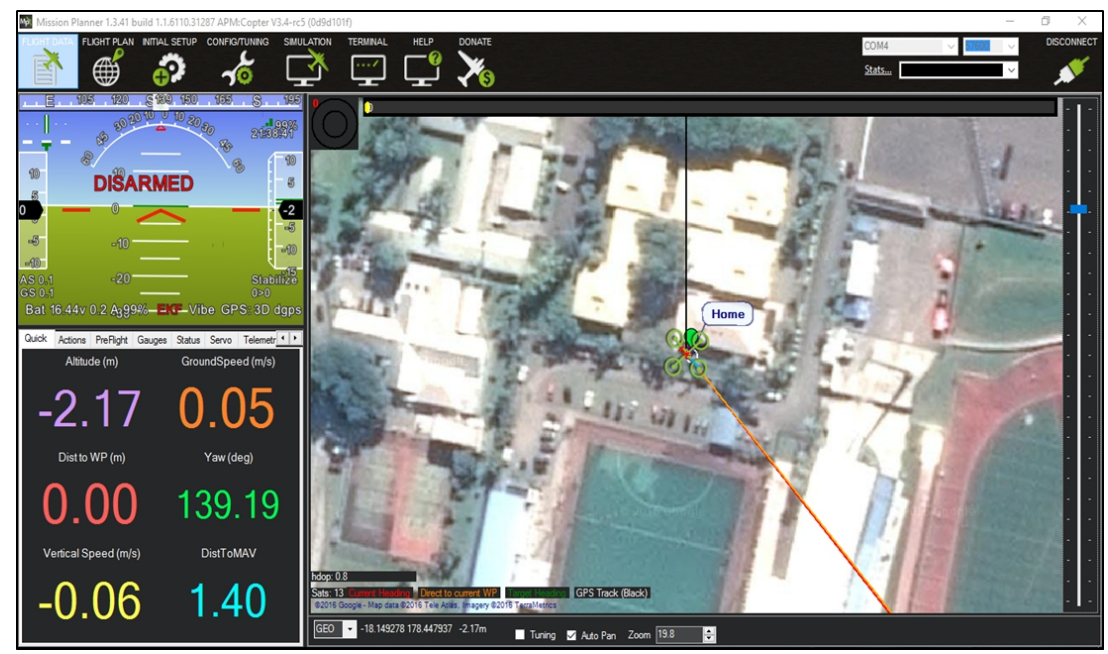

Figure 4. MP view with remote telemetry

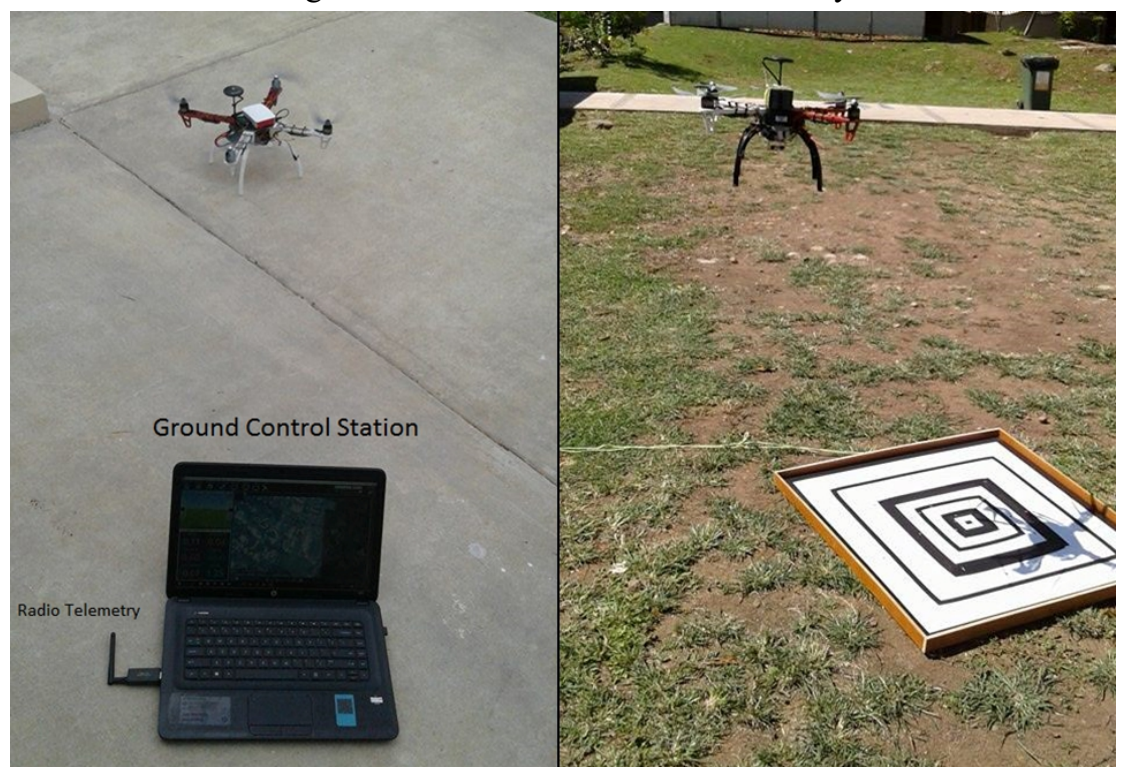

Figure 5. GCS view with quadcopter system

\section{Verification and Discussion}

A precision landing feature and moving target identification while camera and platform both not stationary, the optimization and image processing has been played a major role in this work. An important code considering image processing and target detection is provided in Appendix. The developed system has been tested in real environmental conditions. Tests were conducted in outside open environment, under windy, cloudy, sunny weather conditions. Table 1 shows the accuracy and repeatability of the quadcopter landing. Firstly, it is obvious that we do consider a stationary platform, then also a moving landing platform. However, it is to note that a moving platform has to move at a 
speed less than $1 \mathrm{~m} / \mathrm{s}$ and stop when the quadcopter is hovering $2 \mathrm{~m}$ away. From Table 1 it can be observed that the accuracy of the landing is very favorable and so is its repeatability percentage. From the 24 flight tests, only three went off-platform and one was due to low battery thus the repeatability of the landing is $87 \%$ and the average accuracy is $21.2 \mathrm{~cm}$ considering only the successful land tests.

The distance from the center of the landing platform to the center of the camera is the measured off-set distance. The test flights were done in various weather conditions and these are some of the observations made:

1. In windy and cloudy, it was observed that the quadcopter trajectory was affected by the wind speed at the final landing stage of the flight. There were problems regarding the stability of the quadcopter during the touchdown stage as not all four landing legs hit the landing platform simultaneously and because the motors provided vibrations throughout the body of the quadcopter frame.

Table 1: Quadcopter landing tests

\begin{tabular}{|c|c|}
\hline Flight Number (Testing sequence) & Distance from desired landing location (in cm) \\
\hline 1 & 5 \\
\hline 2 & 13 \\
\hline 3 & 16 \\
\hline 4 & 6.5 \\
\hline 5 & 28 \\
\hline 6 & 4 \\
\hline 7 & 3 \\
\hline 8 & 140 (Off platform) \\
\hline 9 & 28 \\
\hline 10 & 76 \\
\hline 11 & 14.5 \\
\hline 12 & 17 \\
\hline 14 & 26 \\
\hline 15 & 17 \\
\hline 16 & Off platform \\
\hline 17 & 23 \\
\hline 18 & 15 \\
\hline 19 & 19 \\
\hline 20 & 13 \\
\hline 21 & Low Battery \\
\hline 22 & 28 \\
\hline 23 & 44 \\
\hline 24 & Crash \\
\hline
\end{tabular}


2. False detections of square patterns due to varying sun-light conditions.

3. In no wind condition, it landed very close to the center of the landing dock with reduced off-set in terms of the distance from the landing platform center to the center of the camera field of view.

4. In no wind and sunny condition, perfect detection of the landing platform, as shown a very precise landing areas as per flight number 1, 6 and 7 from Table 1.

5. Less movement during the last landing phase of the flight.

Upon changing the version of the camera, the detection performance had improved in both the windy and cloudy conditions. However, the UAV needs to have a perfect GPS lock and a warm-up time usually 5 to 7 minutes before setting out on a flight to help all sensors gauge a stable reading. It is also noted that the internal pressure sensor (barometer) measures atmospheric pressure to gauge the altitude of the quadcopter. However, during flight tests, it was observed from the ground control station that the readings were severely disturbed as even at ground level the altitude readings registered $-2 \mathrm{~m}$ or even $-10 \mathrm{~m}$, varying atmospheric pressure during rainy weather affects the accuracy of the landing process. In addition, it is observed that quadcopters are not to be flown within the vicinity of power lines or large metallic objects as this provides electromagnetic interference to the internal sensors of the PixHawk auto-pilot. It can be observed that the cluster of successful landing points are more concentrated within the first two square boundaries and The landing platform is $86 \times 86 \mathrm{~cm}$ in dimension. In Figure 6 , as the quadcopter moves closer to the landing platform, the concurrent squares detected become less, this is why the squares are concurrently patterned on the landing platform. Thus as the quadcopter moves closer to the landing platform the smaller squares on the concurrent square pattern will be detected. It depicts the quadcopter attempting to center itself over the landing platform and proceed to the final landing stage, in Fig. 5 the video feed from the Pi camera can be seen with the squares on the landing platform detected by the image/video processing program and are being outlined with red lines. 


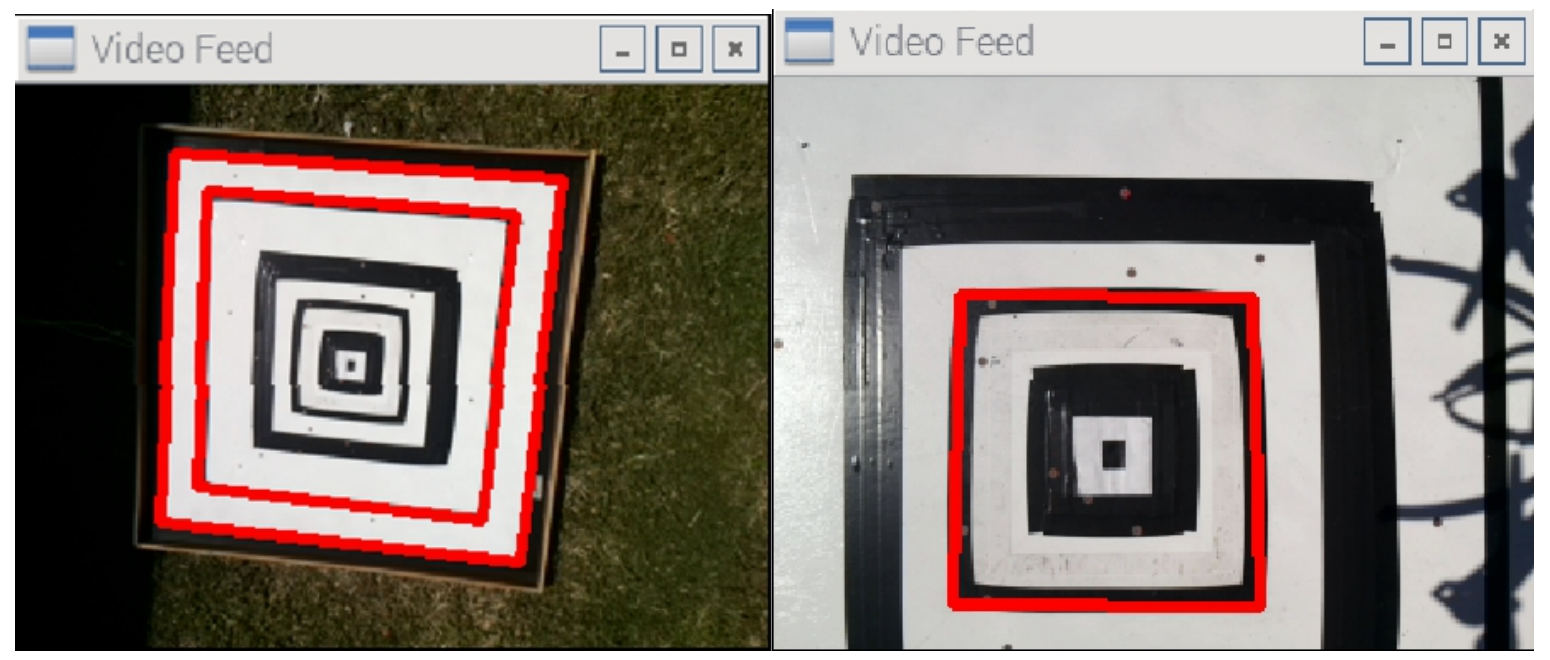

Figure 6. Processing moving platform using camera vision

\section{Conclusion}

The paper has presented the importance of integrating the system with features from Pi-camera and online image processing. The objective of this study was to keep the landing autonomous even when the landing surface is not steady. For the precision landing feature, the quadcopter should utilize both methods of using an IR Beacon together with a Companion Computer. The quadcopter can work for accurate landing given the changing scenarios during the designated landing. Obviously, a further attempt can be made to land on faster moving platforms and handle movements that are more complex, would be future scope. Such application development is very useful for windy and battery-to-fail scenarios. It is observed from result tables that landing accuracy has an average offset of less than 30 $\mathrm{cm}$ with a success rate of $87 \%$. Though landing accuracy is affected by strong winds and weather conditions.

\section{Acknowledgments}

The project team acknowledges the financial support and lab facilities provided during research from the School of Information Technology, Engineering, Mathematics and Physics (STEMP) at the University of the South Pacific, Fiji. Also, Mr. Kevin Rotsaert, Skyward Industries, Nadi for providing the real-time problem and support to verify the efficacy of the result.

\section{References}

1. Aravind, K.R., Raja, P. and Pérez Ruiz, M., 2017. Task-based agricultural mobile robots in arable farming: A review. Spanish Journal of Agricultural Research, 2017 (15 (1)), 1-16.

2. Stodola, P., Drozd, J., Mazal, J., Hodický, J. and Procházka, D., 2019. Cooperative unmanned aerial 
system reconnaissance in a complex urban environment and uneven terrain. Sensors, 19(17), p.3754.

3. Agha-mohammadi, A.A., Ure, N.K., How, J.P. and Vian, J., 2014, September. Health aware stochastic planning for persistent package delivery missions using quadrotors. In 2014 IEEE/RSJ International Conference on Intelligent Robots and Systems (pp. 3389-3396). IEEE.

4. Yao, P., Wang, H. and Ji, H., 2017. Gaussian mixture model and receding horizon control for multiple UAV search in complex environment. Nonlinear Dynamics, 88(2), pp.903-919.

5. Guo, Y., Guo, J., Liu, C., Xiong, H., Chai, L. and He, D., 2020. Precision Landing Test and Simulation of the Agricultural UAV on Apron. Sensors, 20(12), p.3369.

6. Mazzia, V., Comba, L., Khaliq, A., Chiaberge, M. and Gay, P., 2020. UAV and machine learning based refinement of a satellite-driven vegetation index for precision agriculture. Sensors, 20(9), p.2530.

7. Kloepper, L.N. and Kinniry, M., 2018. Recording animal vocalizations from a UAV: bat echolocation during roost re-entry. Scientific reports, 8(1), pp.1-6.

8. Yang, K., Yang, G.Y. and Fu, S.I.H., 2020. Research of control system for plant protection UAV based on pixhawk. Procedia Computer Science, 166, pp.371-375.

9. Ebeid, E., Skriver, M., Terkildsen, K.H., Jensen, K. and Schultz, U.P., 2018. A survey of open-source UAV flight controllers and flight simulators. Microprocessors and Microsystems, 61, pp.11-20.

10. Surastyo, F. and Wirawan, A., 2015. Feasibility Study Implementasi Autopilot Risc Berbasis Arsitektur ARM Sebagai Alternatif Pengganti Autopilot Berbasis Arsitektur AVR. Bunga Rampai Hasil Litbangyasa: Teknologi Pada Pesawat Terbang, Roket, dan Satelit Th 2015, pp.93-100.

11. Sadhu, V., Zonouz, S. and Pompili, D., 2020, May. On-board Deep-learning-based Unmanned Aerial Vehicle Fault Cause Detection and Identification. In 2020 IEEE International Conference on Robotics and Automation (ICRA) (pp. 5255-5261). IEEE.

12. Nemes, A., 2015. Synopsis of soft computing techniques used in quadrotor UAV modelling and control. Interdisciplinary Description of Complex Systems: INDECS, 13(1), pp.15-25.

13. Anitha, G. and Kumar, R.G., 2012. Vision based autonomous landing of an unmanned aerial vehicle. Procedia Engineering, 38, pp.2250-2256.

14. Mehta, U., Alim, M. and Kumar, S., 2017. Smart path guidance mobile aid for visually disabled persons. Procedia Computer Science, 105, pp.52-56.

15. Kumar, R., Mehta, U. and Chand, P., 2017. A low cost linear force feedback control system for a two-fingered parallel configuration gripper. Procedia Computer Science, 105, pp.264-269.

16. Sharma, B., Vanualailai, J. and Singh, S., 2017. Motion planning and posture control of multiple n-link doubly nonholonomic manipulators. Robotica, 35(1), pp.1-25.

17. Majhi, S., Kotwal, V., Mehta, U., 2012, FPAA-Based PI controller for DC servo position control system. IFAC Proceedings Volumes, 45(3), pp. 247-251.

18. Guo, D., Wang, Y., Zhong, M. and Zhao, Y., 2018. Fault detection and isolation for Unmanned Aerial Vehicle sensors by using extended PMI filter. IFAC-PapersOnLine, 51(24), pp.818-823.

19. Alexis, K., Nikolakopoulos, G. and Tzes, A., 2012. Model predictive quadrotor control: attitude, altitude and position experimental studies. IET Control Theory \& Applications, 6(12), pp.1812-1827.

20. Feng, Y., Zhang, C., Baek, S., Rawashdeh, S. and Mohammadi, A., 2018. Autonomous landing of a UAV on a moving platform using model predictive control. Drones, 2(4), p.34.

21. Guo, Y., Wang, J., Chen, H., Li, G., Huang, R., Yuan, Y., Ahmad, T. and Sun, S., 2019. An expert rule-based fault diagnosis strategy for variable refrigerant flow air conditioning systems. Applied Thermal Engineering, 149, pp.1223-1235.

22. Yi, Z. and Etemadi, A.H., 2016. Fault detection for photovoltaic systems based on multi-resolution signal decomposition and fuzzy inference systems. IEEE Transactions on Smart Grid, 8(3), 
pp.1274-1283.

23. Chen, Y., Zhen, Z., Yu, H. and Xu, J., 2017. Application of fault tree analysis and fuzzy neural networks to fault diagnosis in the internet of things (IoT) for aquaculture. Sensors, 17(1), p.153.

24. Olivares-Mendez, M.A., Kannan, S. and Voos, H., 2015, June. Vision based fuzzy control autonomous landing with UAVs: From V-REP to real experiments. In 2015 23rd Mediterranean Conference on Control and Automation (MED) (pp. 14-21). IEEE.

25. Tweedale, J.W., 2012, June. Fuzzy control loop in an autonomous landing system for unmanned air vehicles. In 2012 IEEE International Conference on Fuzzy Systems (pp. 1-8). IEEE.

26. Chen, F., Jiang, R., Zhang, K., Jiang, B. and Tao, G., 2016. Robust backstepping sliding-mode control and observer-based fault estimation for a quadrotor UAV. IEEE Transactions on Industrial Electronics, 63(8), pp.5044-5056.

27. Cherubini, A., Papini, A., Vertechy, R. and Fontana, M., 2015. Airborne Wind Energy Systems: A review of the technologies. Renewable and Sustainable Energy Reviews, 51, pp.1461-1476.

28. Williams, A. and Yakimenko, O., 2018, April. Persistent mobile aerial surveillance platform using intelligent battery health management and drone swapping. In 2018 4th International Conference on Control, Automation and Robotics (ICCAR) (pp. 237-246). IEEE.

29. Sri, K.R.B., Aneesh, P., Bhanu, K. and Natarajan, M., 2016. Design analysis of solar-powered unmanned aerial vehicle. Journal of Aerospace Technology and Management, 8(4), pp.397-407.

30. Guedim, Z. Hydrogen-Powered Drone 'Hycopter' Stays in Flight for 4 Hours. 2018. Available online: Edgylabs.com/hydrogen-powered-drone-hycopter-flight-4-hours (accessed on 15 April 2021).

31. Herissé, B., Hamel, T., Mahony, R. and Russotto, F.X., 2011. Landing a VTOL unmanned aerial vehicle on a moving platform using optical flow. IEEE Transactions on robotics, 28(1), pp.77-89.

32. Kong, W., Zhang, D., Wang, X., Xian, Z. and Zhang, J., 2013, November. Autonomous landing of an UAV with a ground-based actuated infrared stereo vision system. In 2013 IEEE/RSJ International Conference on Intelligent Robots and Systems (pp. 2963-2970). IEEE. 